\title{
Asymptotic preserving schemes for the Wigner-Poisson-BGK equations in the diffusion limit
}

\author{
Nicolas Crouseilles * Giovanni Manfredi ${ }^{\dagger}$
}

November 4, 2012

\begin{abstract}
This work focusses on the numerical simulation of the Wigner-Poisson-BGK equation in the diffusion asymptotics. Our strategy is based on a "micro-macro" decomposition, which leads to a system of equations that couple the macroscopic evolution (diffusion) to a microscopic kinetic contribution for the fluctuations. A semi-implicit discretization provides a numerical scheme which is stable with respect to the small parameter $\varepsilon$ (mean free path) and which possesses the following properties: (i) it enjoys the asymptotic preserving property in the diffusive limit; (ii) it recovers a standard discretization of the Wigner-Poisson equation in the collisionless regime. Numerical experiments confirm the good behaviour of the numerical scheme in both regimes. The case of a spatially dependent $\varepsilon(x)$ is also investigated.
\end{abstract}

\section{Introduction}

The ongoing miniaturization of microelectronics devices makes classical transport models (e.g., the drift-diffusion equation) unable to capture the main features of a variety of systems - such as semiconductor quantum dots and resonant tunneling diodes - where quantum mechanical effects are expected to play a central role. This fact motivates the development of quantum transport models for charged particle systems.

The Wigner representation $[28,12]$ is a useful tool to express quantum mechanics in a phase space formalism. In this representation, a quantum state is described by a Wigner function (i.e., a function of the phase space variables), whose temporal evolution obeys an integro-differential equation (Wigner equation) that is similar to the classical Liouville equation. The Wigner function cannot be regarded as a true probability density in the phase space, as it may take negative values. Nevertheless, it can be used to compute averages just like in classical statistical mechanics.

The Wigner equation can be coupled to the Poisson equation for the electrostatic potential to obtain a suitable model to describe the quantum dynamics of electrons [22, 27]. Such selfconsistent Wigner-Poisson equations have been extensively used to study quantum transport in semiconductors and metallic nanostructures, such as nanoparticles and thin films [11, 26].

In semiconductor devices, the electron motion is coupled to the ion lattice vibrations (phonons) which act as a thermal bath driving the electron population towards classical equilibrium on a timescale $\tau$ [26]. Therefore, in order to deal with a wide range of physical regimes, it is important to construct a numerical model that is stable and accurate both in the fully quantum regime $(\tau$ large compared to some typical timescale) and in the classical collision-dominated regime ( $\tau$ small).

\footnotetext{
*Inria Rennes-Bretagne Atlantique, IPSO Project \& IRMAR (UMR 6625) Université de Rennes 1, France

${ }^{\dagger}$ Institut de Physique et Chimie des Matériaux de Strasbourg, Université de Strasbourg and CNRS, UMR 7504, F-67034 Strasbourg, France
} 
In the present context, the classical limit model is the drift-diffusion (DD) equation, corresponding to the limit $\tau \approx \varepsilon \rightarrow 0$ in the scaled Wigner equation (2.7). Our purpose is to design an efficient asymptotic-preserving (AP) scheme for the diffusion asymptotics of the Wigner equation. The term "asymptotic preserving" has been introduced in [13] for numerical schemes that are stable with respect to a small parameter $\varepsilon$ and degenerate into a consistent numerical scheme for the limit model when $\varepsilon \rightarrow 0$.

The diffusion limit has already been studied for the radiative transfer equation or the collisional Vlasov equation - we refer to Refs. $[14,7,20,15,16,18,19,21]$ for further details. Other asymptotic limits, such as the fluid limit or the high field limit, have been investigated in Refs. [4, 10, 8, 17].

In this work, we study the diffusion limit of the Wigner equation using the so-called "micromacro" decomposition $[4,25,23,8]$. This strategy seems to be a robust and systematic way to design AP schemes. The main idea is to develop a micro-macro model (equivalent to the original Wigner equation) which couples the macroscopic part of the evolution to a microscopic part. A suitable semi-implicit discretization then ensures that the corresponding numerical scheme satisfies the asymptotic-preserving property. However, in the collisionless limit $(\varepsilon \rightarrow \infty)$, such a scheme displays a modified time discretization of the original Wigner-Poisson model, which may affect the long-time behaviour of the solution. Hence, we introduce a slight improvement that ensures a standard time discretization of the Wigner-Poisson equation in the collisionless limit without affecting the asymptotic-preserving character of the numerical scheme.

We shall restrict our analysis to one-dimensional (1D) problems and consider the Wigner distribution function $f(t, x, v)$, which depends on time $t \geq 0$, space $x \in[0, L]$ and velocity $v \in \mathbb{R}$. Denoting by $\phi(t, x)$ the self-consistent electric potential $\left(E=-\partial_{x} \phi\right.$ is the electric field), the collisional Wigner equation reads as (with $e$ and $m$ represent the electron charge and mass):

$$
\frac{\partial f}{\partial t}+v \partial_{x} f-\frac{e}{m} T_{\hbar / m}[\phi] f=\frac{1}{\tau} Q(f)
$$

where $\tau$ is a relaxation time and

$$
\left(T_{\hbar / m}[\phi] f\right)(t, x, v)=\frac{\mathrm{i}}{2 \pi} \int_{\mathbb{R}_{\xi}} \int_{\mathbb{R}_{v^{\prime}}} \frac{\phi\left(t, x+\frac{\hbar}{2 m} \xi\right)-\phi\left(t, x-\frac{\hbar}{2 m} \xi\right)}{\hbar / m} f\left(t, x, v^{\prime}\right) \exp \left[-\mathrm{i}\left(v-v^{\prime}\right) \xi\right] d v^{\prime} d \xi,
$$

with $\hbar$ the Planck constant. The self-consistent potential obeys the Poisson equation

$$
\partial_{x}^{2} \phi(t, x)=-e\left[\rho(t, x)-n_{i}(x)\right]
$$

where $\rho(t, x)=\int_{\mathbb{R}} f(t, x, v) d v$ and $n_{i}$ is a given ion density. The collision operator $Q(f)$ is the Bhatnagar-Gross-Krook (BGK) collision operator

$$
Q(f)(t, x, v)=\rho(t, x) M_{\Theta}(v)-f(t, x, v), \quad \text { with } \quad M_{\Theta}(v)=\frac{1}{\sqrt{2 \pi \Theta}} \exp \left(-\frac{v^{2}}{2 \Theta}\right),
$$

with $\Theta=k_{B} T / m, k_{B}$ being the Boltzmann constant and $T$ a given temperature.

We consider a spatially periodic plasma with period $L$. The distribution function $f$ satisfies the following boundary conditions

$$
f(t, 0, v)=f(t, L, v), \quad \forall v \in \mathbb{R}, \quad t \geq 0 .
$$

In order to obtain a well-posed problem, a zero-mean condition has to be imposed on the electric field:

$$
\int_{0}^{L} E(t, x) d x=0, \quad \forall t \geq 0
$$


Finally, an initial condition should be specified

$$
f(0, x, v)=f_{0}(x, v), \quad \forall x \in[0, L], v \in \mathbb{R} .
$$

The rest of the paper is organized as follows. In Sec. 2, the diffusive scaling and the corresponding asymptotics are presented in the Wigner-BGK case. In Sec. 3, a linear stability analysis is performed and the relevant growth rate are computed. In Sec. 4, we present the asymptoticpreserving numerical scheme for the Wigner-BGK equation in the diffusive scaling. The results of several numerical tests are shown in Sec. 5, and conclusions are finally drawn in Sec. 6 .

\section{Diffusion regime for Wigner-BGK}

In this section, we detail the diffusion scaling for (1.1) and the corresponding asymptotic model (we refer to [1] for more details).

\subsection{Diffusion scaling}

We normalize space to a characteristic macroscopic length $\ell$, velocity to the thermal speed $\sqrt{\Theta}$, and time to $\ell / U_{0}$, where $U_{0}$ is a typical drift velocity. The electric potential is normalized to $m \Theta / e$ and the electron density to the average density $\rho_{0}$. We also assume that the ion density profile is uniform, i.e., $n_{i}(x)=\rho_{0}$.

With these normalizations, the Wigner-BGK equation becomes

$$
\eta \partial_{t} f+v \partial_{x} f-T_{\nu h_{0}}[\phi] f=\frac{1}{\nu}\left(\rho M_{\Theta}-f\right),
$$

and the Poisson equation can be written as

$$
\partial_{x}^{2} \phi=-\gamma(\rho-1)
$$

where $\eta=U_{0} / \sqrt{\Theta}, \nu=\sqrt{\Theta} \tau / \ell$, and $\gamma=\rho_{0} e^{2} \ell^{2} /\left(m \Theta \epsilon_{0}\right)=\omega_{p}^{2} \ell^{2} / \Theta=\ell^{2} / \lambda_{D}^{2}$, where $\lambda_{D}$ is the Debye length. In the forthcoming simulations, we will always take $\gamma=1$.

In the diffusion regime, we assume that $\eta=\nu=\varepsilon \ll 1$, which means that the drift velocity $U_{0} \sim \varepsilon$ is small compared to the thermal velocity $\sqrt{\Theta} \sim 1$, which in turn is small compared to the relaxation velocity $\ell / \tau \sim \varepsilon^{-1}$. The relevant ordering can thus be written as

$$
U_{0} \ll \sqrt{\Theta} \ll \frac{\ell}{\tau} .
$$

With the above scaling, the Wigner equation becomes

$$
\varepsilon \partial_{t} f+v \partial_{x} f-T_{\varepsilon h_{0}}[\phi] f=\frac{1}{\varepsilon}\left(\rho M_{\Theta}-f\right),
$$

where the normalized Planck constant $h_{0}$ is defined as

$$
h_{0}=\frac{\hbar}{m \Theta \tau},
$$

and

$$
T_{\varepsilon h_{0}}[\phi] f=\frac{\mathrm{i}}{2 \pi} \int_{\mathbb{R}_{\xi}} \int_{\mathbb{R}_{v^{\prime}}} \frac{\phi\left(t, x+\frac{\varepsilon h_{0}}{2} \xi\right)-\phi\left(t, x-\frac{\varepsilon h_{0}}{2} \xi\right)}{\varepsilon h_{0}} f\left(t, x, v^{\prime}\right) \exp \left[-\mathrm{i}\left(v-v^{\prime}\right) \xi\right] d v^{\prime} d \xi .
$$




\subsection{Derivation of the asymptotic drift-diffusion model}

To derive the DD model from Eq. (2.7) in the asymptotic limit $\varepsilon \rightarrow 0$, the usual procedure is to perform a Chapman-Enskog expansion of the Wigner function: $f=f_{0}+\varepsilon f_{1}+\varepsilon^{2} f_{2}$. This expansion is injected in (2.7), and the terms of same order are identified. At the lowest order $\varepsilon^{-2}$, we obtain

$$
f_{0}(t, x, v)=\rho(t, x) M_{\Theta}(v), \quad \rho(t, x)=\int f_{0}(t, x, v) d v .
$$

In particular, the first moment of $f$ is equal to the first moment of $f_{0}$; as a consequence, the first moment of $f_{1}$ and $f_{2}$ is equal to zero. Identifying terms of order $\varepsilon^{-1}$, we find that $f_{1}$ has to satisfy

$$
v \partial_{x} f_{0}-\partial_{x} \phi \partial_{v} f_{0}=-f_{1} .
$$

This equation has a solution if and only if the right hand side satisfies the null average condition:

$$
\int\left(v \partial_{x} f_{0}-\partial_{x} \phi \partial_{v} f_{0}\right) d v=0
$$

Using the relation $f_{0}=\rho M_{\Theta}$, we have $\int_{\mathbb{R}} v \partial_{x} f_{0} d v=0$, and $\int_{\mathbb{R}} \partial_{x} \phi \partial_{v} f_{0} d v=0$. Hence, the integral of the right hand side of (2.8) is equal to zero and (2.8) has a unique solution $f_{1}$ given by

$$
f_{1}=-v \partial_{x} f_{0}+\partial_{x} \phi \partial_{v} f_{0}
$$

Finally, keeping terms of order unity leads to

$$
\partial_{t} f_{0}+v \partial_{x} f_{1}-T_{\varepsilon h_{0}}[\phi] f_{1}=Q\left(f_{2}\right)=-f_{2} .
$$

The solvability condition for $f_{2}$ implies that

$$
\partial_{t} \rho+\partial_{x}\left(\int_{\mathbb{R}} v f_{1} d v\right)=0
$$

since $\int T_{\varepsilon h_{0}}[\phi] f_{1} d v=0$. Using (2.9), we get

$$
\int_{\mathbb{R}} v f_{1} d v=-\partial_{x} \int_{\mathbb{R}} v^{2} f_{0} d v+\int_{\mathbb{R}} v \partial_{x} \phi \partial_{v} f_{0} d v=-\partial_{x}(\rho \Theta)-\rho \partial_{x} \phi,
$$

so that (2.10) becomes the so-called DD equation

$$
\partial_{t} \rho-\partial_{x}\left[\rho \partial_{x} \phi+\partial_{x}(\rho \Theta)\right]=0 .
$$

\subsection{Derivation of the micro-macro model and the asymptotic limit}

This section is devoted to the derivation of the micro-macro model starting from the Wigner-BGK equation (see [4, 8, 23, 25]).

\section{Derivation of the micro-macro model}

Let us suppose that $f$ satisfies the following decomposition (we assume $\Theta=1$ for simplicity)

$$
f=\rho M+g, \text { with } M(v)=\frac{1}{\sqrt{2 \pi}} \exp \left(-\frac{v^{2}}{2}\right), \quad \rho=\int f d v .
$$

We introduce the transport operator $\mathcal{T} f=v \partial_{x} f-T_{\varepsilon h_{0}}[\phi] f$ of the Wigner equation (1.1), which leads to

$$
\partial_{t}(\rho M)+\partial_{t} g+\frac{1}{\varepsilon} \mathcal{T}(\rho M)+\frac{1}{\varepsilon} \mathcal{T} g=-\frac{1}{\varepsilon^{2}} g .
$$


We define $\Pi$ as the orthogonal projection in $L^{2}\left(M^{-1} d v\right)$, endowed with the weighted scalar product $(\varphi, \psi)_{M}=\left\langle\varphi \psi M^{-1}\right\rangle=\int \varphi \psi d v / M$, onto the kernel of the collision operator

$$
\mathcal{N}(Q)=\left\{f=\rho M \text { where } \rho=\int f d v\right\} .
$$

For any function $\varphi$ depending on $v$, the explicit orthogonal projection onto the kernel is given by

$$
\Pi(\varphi)=\int \varphi d v M
$$

In this case, the projection is nothing but an integration in $v$ multiplied by the a Maxwellian function. But this approach is general and can be adapted to different cases (see [4, 8, 23, 25, 24]).

The micro-macro model is obtained by applying $\Pi$ and $(I-\Pi)$ on (2.12). Applying $\Pi$ to $(2.12)$ provides an equation for $\rho$, whereas applying $(I-\Pi)$ gives an equation for $g$. The micro-macro model of unknown $(g, U, \phi)$ can then be written

$$
\left\{\begin{array}{rlrl}
\partial_{t} g+\frac{1}{\varepsilon}(I-\Pi) \mathcal{T} g & =\frac{1}{\varepsilon^{2}}[-g-\varepsilon(I-\Pi) \mathcal{T}(\rho M)], & & \text { "micro" } \\
\partial_{t} \rho+\frac{1}{\varepsilon} \partial_{x} \int v g d v & =0, & & \text { "macro" } \\
\partial_{x}^{2} \phi & =-(\rho-1), & \text { "Poisson" }
\end{array}\right.
$$

where $\Pi$ is defined in (2.13), $\mathcal{T} g=v \partial_{x} g-T_{\varepsilon h_{0}}[\phi] g$ and $M(v)=(1 / \sqrt{2 \pi}) \exp \left(-v^{2} / 2\right)$.

Proposition 2.1. (i) If $(f, \phi)$ is a solution of (1.1)-(1.2)-(1.3) with the initial data (1.6), then $(\rho, g, \phi)=\left(\int f d v, f-\rho M, \phi\right)$ is a solution of (2.14) with the associated initial data

$\rho(t=0)=\int f(t=0) d v, \quad g(t=0)=f(t=0)-\rho(t=0) M, \quad$ and $\quad-\partial_{x}^{2} \phi(t=0)=\rho(t=0)-1$.

(ii) Conversely, if $(\rho, g, \phi)$ is a solution of (2.14) with initial data (2.15), then $\int g d v=0$ and $f=\rho M+g$ is a solution of (1.1)-(1.2)-(1.3).

\section{Chapman-Enskog expansion}

In this paragraph, we want to verify formally that the asymptotic limit of (2.14) is indeed the DD model (2.11) derived previously. Multiplying the first equation of (2.14) by $\varepsilon^{2}$, we deduce that $g=\mathcal{O}(\varepsilon)$ so that we can write from the first equation of $(2.14)$,

$$
\begin{aligned}
g & =-\varepsilon(I-\Pi) \mathcal{T}(\rho M)+\mathcal{O}\left(\varepsilon^{2}\right) \\
& =-\varepsilon(I-\Pi)\left(v M \partial_{x} \rho-T_{\varepsilon h_{0}}[\phi](\rho M)\right)+\mathcal{O}\left(\varepsilon^{2}\right), \\
& =-\varepsilon(I-\Pi)\left(v M \partial_{x} \rho-\partial_{x} \phi \partial_{v}(\rho M)+\mathcal{O}\left(\varepsilon^{2}\right)\right. \\
& =-\varepsilon\left(v M \partial_{x} \rho-\partial_{x} \phi \partial_{v}(\rho M)\right)+\mathcal{O}\left(\varepsilon^{2}\right) \\
& =-\varepsilon\left(v M \partial_{x} \rho+v \rho M \partial_{x} \phi\right)+\mathcal{O}\left(\varepsilon^{2}\right) .
\end{aligned}
$$

If we now inject the above expression into the second equation of $(2.14)$, we obtain

$$
\partial_{t} \rho-\partial_{x}\left[\int v^{2} M d v \partial_{x} \rho+\rho \partial_{x} \phi\right]=\mathcal{O}\left(\varepsilon^{2}\right) .
$$

or, when $\varepsilon$ goes to zero,

$$
\partial_{t} \rho-\partial_{x}\left[\partial_{x} \rho+\rho \partial_{x} \phi\right]=0
$$

which is exactly the drift-diffusion model (2.11).

These basic computations will be mimicked at the discrete level. 


\section{$3 \quad$ Linear stability analysis}

In this section, we study the linear response of the Wigner-BGK equations in order to validate the collisionless limit [i.e., $\varepsilon \rightarrow \infty$ in (2.7)] in the numerical simulations.

In order to investigate the linear response of the Wigner-Poisson equations, we expand the distribution function and the potential around the equilibrium solution $f=f_{0}(v), \phi=0$ :

$$
f(t, x, v)=f_{0}(v)+f_{1}(t, x, v), \quad \phi(t, x)=\phi_{1}(t, x),
$$

and then neglect second order terms. Further, we assume that the perturbed quantities can be expanded in a Fourier series both in space and in time, i.e.:

$$
\begin{aligned}
f_{1}(x, v, t) & =\widetilde{f}_{1}(v) \exp (-i \omega t+i k x), \\
\phi_{1}(x, t) & =\widetilde{\phi_{1}} \exp (-i \omega t+i k x),
\end{aligned}
$$

where $\omega$ and $k$ are the frequency and wave number of the perturbation.

We first apply this approach to the Wigner-Poisson equations without the BGK term, which disappears in the limit $\varepsilon \rightarrow \infty$. In this case, the relevant dispersion relation can be written in terms of the Lindhardt "dielectric constant" [2]

$$
D(\Omega, k) \equiv 1+\frac{1}{H k^{2}} \int_{-\infty}^{+\infty} \frac{f_{0}(v+H k / 2)-f_{0}(v-H k / 2)}{\Omega-k v} d v=0
$$

where we have defined $\Omega=\omega \varepsilon$ and $H=h_{0} \varepsilon$.

Expanding in a Taylor series the difference term in the integral in Eq. (3.18), we obtain

$$
D(\Omega, k) \approx 1+\frac{1}{k} \int \frac{f_{0}^{\prime}(v)}{\Omega-k v} d v+\frac{H^{2} k}{24} \int \frac{f_{0}^{\prime \prime \prime}(v)}{\Omega-k v} d v+\ldots
$$

where the apex denotes derivation with respect to $v$. Notice that the first two terms are identical to the dispersion relation for the Vlasov-Poisson equations, whereas the last term is a quantum correction.

We further assume that the wave number of the perturbation is small, i.e., $k v \ll \Omega$, and use this fact to write the following expansion:

$$
\frac{1}{\Omega-k v} \approx \frac{1}{\Omega}+\frac{k v}{\Omega^{2}}+\frac{k^{2} v^{2}}{\Omega^{3}}+\frac{k^{3} v^{3}}{\Omega^{4}}+\ldots
$$

If the equilibrium distribution $f_{0}(v)$ is an even function of $v$, the dielectric constant can then be expressed in the following way:

$$
D(\Omega, k) \approx 1-\frac{1}{\Omega^{2}}-\frac{3 k^{2}\left\langle v^{2}\right\rangle}{\Omega^{4}}-\frac{H^{2} k^{4}}{4 \Omega^{4}}
$$

where $\left\langle v^{2}\right\rangle:=\int v^{2} f_{0}(v) d v$, or, equivalently to the relevant order

$$
D(\Omega, k)=1-\left\{\Omega^{2}-3 k^{2} \theta-\frac{H^{2} k^{4}}{4}\right\}^{-1}
$$

where we have used the fact that, for a Maxwellian distribution $f_{0}(v)=M_{\theta}(v)$, we have $\left\langle v^{2}\right\rangle=\theta$.

Now, for an equilibrium distribution shifted of a constant velocity $\pm V$, the above dispersion relation becomes simply:

$$
D_{ \pm}(\Omega, k)=1-\left\{(\Omega \pm k V)^{2}-3 k^{2} \theta-\frac{H^{2} k^{4}}{4}\right\}^{-1} .
$$


In the forthcoming sections, we will be interested in two-stream distributions composed of two Maxwellians traveling with velocities $\pm V$. In that case, the dielectric constant can be written as

$$
D(\Omega, k)=\frac{D_{+}(\Omega, k)+D_{-}(\Omega, k)}{2} .
$$

Setting $D(k, \omega)=0$, we obtain the dispersion relation for the two-stream plasma

$$
\begin{aligned}
\Omega^{4} & -\left(1+2 k^{2}\left(V^{2}+3 \theta\right)+\frac{H^{2} k^{4}}{2}\right) \Omega^{2} \\
& -k^{2}\left(V^{2}-3 \theta-\frac{H^{2} k^{2}}{4}\right)\left(1-\left(V^{2}-3 \theta\right) k^{2}+\frac{H^{2} k^{4}}{4}\right)=0 .
\end{aligned}
$$

Solving for $\Omega^{2}$, one obtains

$$
\Omega^{2}=\frac{1}{2}+k^{2}\left(V^{2}+3 \theta+\frac{H^{2} k^{2}}{4}\right) \pm \frac{1}{2}\left[1+8 k^{2}\left(V^{2}+6 k^{2} \theta+\frac{H^{2} k^{4}}{2}\right)\right]^{1 / 2} .
$$

Instability occurs when $\Omega^{2}<0$, and the instability rate is simply given by $\operatorname{Im}(\Omega) / \varepsilon$.

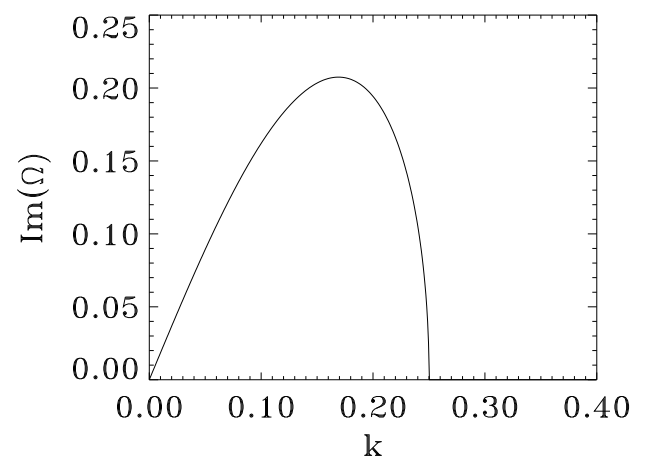

Figure 1: Plot of the instability rate for a two-stream distribution, $h_{0}=0.01, \varepsilon=1000, V=2$, $\theta=0.2$.

\section{Numerical approximation}

In the following, we shall use the notation $\mathcal{T} f=v \partial_{x} f-T_{\varepsilon h_{0}}\left[\phi^{n}\right] f$ (the electric potential is evaluated at time $t^{n}$ ).

\subsection{Time discretizations}

\section{Semi-implicit time discretization}

Following $[25,8]$, the main idea is to treat the stiffest terms in an implicit way. In the micro equation, the stiffest term is $g / \varepsilon^{2}$, whereas in the macro equation the flux $\int v g d v$ should also be treated implicitly. We then get the following semi-discretization in time for (2.14)

$$
\left\{\begin{aligned}
\frac{g^{n+1}-g^{n}}{\Delta t}+\frac{1}{\varepsilon}(I-\Pi) \mathcal{T} g^{n} & =\frac{1}{\varepsilon^{2}}\left[-g^{n+1}-\varepsilon(I-\Pi) \mathcal{T}\left(\rho^{n} M\right)\right], \\
\frac{\rho^{n+1}-\rho^{n}}{\Delta t}+\frac{1}{\varepsilon} \partial_{x} \int v g^{n+1} d v & =0 \\
-\partial_{x}^{2} \phi^{n} & =\rho^{n}-1 .
\end{aligned}\right.
$$


The first equation can be rewritten as

$$
g^{n+1}=\left(1+\frac{\varepsilon^{2}}{\Delta t}\right)^{-1}\left(\frac{\varepsilon^{2}}{\Delta t} g^{n}-\varepsilon(I-\Pi) \mathcal{T}\left(\rho^{n} M+g^{n}\right)\right) .
$$

Once $g^{n+1}$ has been computed, the macro equation on $\rho$ can be advanced to get $\rho^{n+1}$ and the electric potential can be solved to obtain $\phi^{n+1}$.

\section{AP property}

Let us verify that the previous numerical scheme provides a consistent scheme for (2.11) when $\varepsilon$ goes to zero. When $\varepsilon \ll 1$, we have from the micro equation that $g^{n+1}=\mathcal{O}(\varepsilon)$. Then, in this regime, we have from $(4.27)$

$$
\begin{aligned}
g^{n+1} & =-\varepsilon(I-\Pi) \mathcal{T}\left(\rho^{n} M\right)+\mathcal{O}\left(\varepsilon^{2}\right), \\
& =-\varepsilon \mathcal{T}\left(\rho^{n} M\right)+\mathcal{O}\left(\varepsilon^{2}\right), \\
& =-\varepsilon\left(v M \partial_{x} \rho^{n}-T_{\varepsilon h_{0}}\left[\phi^{n}\right]\left(\rho^{n} M\right)\right)+\mathcal{O}\left(\varepsilon^{2}\right), \\
& =-\varepsilon\left(v M \partial_{x} \rho^{n}-\partial_{x} \phi^{n} \partial_{v}\left(\rho^{n} M\right)\right)+\mathcal{O}\left(\varepsilon^{2}\right), \\
& =-\varepsilon\left(v M \partial_{x} \rho^{n}+v \rho^{n} M \partial_{x} \phi^{n}\right)+\mathcal{O}\left(\varepsilon^{2}\right),
\end{aligned}
$$

which, injected into the macro equation, leads to

$$
\frac{\rho^{n+1}-\rho^{n}}{\Delta t}-\partial_{x}\left(\int v^{2} M d v \partial_{x} \rho^{n}+\int v^{2} M d v \rho^{n} \partial_{x} \phi^{n}\right)=0 .
$$

Using $\int v^{2} M d v=1$, the above equation can be rewritten as

$$
\frac{\rho^{n+1}-\rho^{n}}{\Delta t}-\partial_{x}\left(\partial_{x} \rho^{n}+\rho^{n} \partial_{x} \phi^{n}\right)=0,
$$

which is an explicit time discretization of the limit model (2.11). Let us also mention that an implicit discretization of the diffusion term $\partial_{x}^{2} \rho$ can be derived using the methods described in Refs. [23, 8].

\section{Mixed scheme}

Here, we want to design a numerical scheme which, in the limit $\varepsilon \rightarrow+\infty$, recovers an explicit numerical scheme for the Wigner-Poisson equation

$$
f^{n+1}=f^{n}-\frac{\Delta t}{\varepsilon} \mathcal{T} f^{n}+\mathcal{O}\left(\frac{1}{\varepsilon^{2}}\right)
$$

which was not the case for the above time discretization. Indeed, adding together the first two equations of (4.26) leads to

$$
f^{n+1}=f^{n}-\frac{\Delta t}{\varepsilon} \mathcal{T} f^{n}-\frac{\Delta t}{\varepsilon} \Pi\left(v \partial_{x}\left(g^{n+1}-g^{n}\right)\right)+\mathcal{O}\left(\frac{1}{\varepsilon^{2}}\right),
$$

which involves an additional term of order $\mathcal{O}\left(\Delta t^{2}\right)$ that can pollute the long-time behaviour of the numerical solution.

On the other hand, an explicit (but not stable) discretization of the macro equation gives

$$
\rho^{n+1}=\rho^{n}-\frac{\Delta t}{\varepsilon} \int \mathcal{T} f^{n} d v
$$


where $f^{n}=\rho^{n} M+g^{n}$. Multiplying Eq. (4.30) by $M$ and adding to the first of equations (4.26) for $g$, yields

$$
f^{n+1}=f^{n}-\frac{\Delta t}{\varepsilon} \mathcal{T} f^{n}+\mathcal{O}\left(\frac{1}{\varepsilon^{2}}\right)
$$

If the transport dominates over the dissipation phenomena (which occurs when $\varepsilon \rightarrow+\infty$ ), then one would like to recover this discretization of the initial kinetic equation.

One way to derive a numerical scheme that is well behaved both when $\varepsilon \rightarrow 0$ and when $\varepsilon \rightarrow \infty$, is to combine the flux in (4.30) with the flux $\Pi\left(v \partial_{x} g^{n+1}\right)$. To that purpose, we choose a linear combination of these two fluxes

$$
\rho^{n+1}=\rho^{n}-\beta \frac{\Delta t}{\varepsilon} \int \mathcal{T} f^{n} d v-(1-\beta) \frac{\Delta t}{\varepsilon} \partial_{x} \int\left(v g^{n+1}\right) d v
$$

where we require $\beta$ to satisfy the following properties:

- $\beta \in[0,1]$, for the consistency;

- $\beta=\mathcal{O}\left(\varepsilon^{2}\right)$ when $\varepsilon \rightarrow 0$, to preserve the AP property;

- $\lim _{\varepsilon \rightarrow \infty} \beta=1$ to ensure the correct collisionless limit when $\varepsilon \rightarrow+\infty$.

One good choice appears to be $\beta=\varepsilon^{2} /\left(1+\varepsilon^{2}\right)$.

Hence, we still keep the consistency with respect to the initial model for a fixed $\varepsilon>0$ since we have used a linear combination of two consistent fluxes with $\beta \in[0,1]$. Moreover, when $\varepsilon \rightarrow 0$, we have $\beta / \varepsilon \rightarrow 0$ so that we recover the previous stable discretization

$$
\rho^{n+1}=\rho^{n}-\frac{\Delta t}{\varepsilon} \partial_{x} \int\left(v g^{n+1}\right) d v+\mathcal{O}(\varepsilon),
$$

which is consistent with the limit drift-diffusion model (see above). On the other hand, when $\varepsilon \rightarrow+\infty$, we have $\beta \rightarrow 1$ so that we recover

$$
\rho^{n+1}=\rho^{n}-\frac{\Delta t}{\varepsilon} \int \mathcal{T} f^{n} d v+\mathcal{O}\left(\frac{1}{\varepsilon^{2}}\right)
$$

which, added to the equation for $g$ [i.e., the first equation of (4.26)] leads to the explicit discretization (4.29) of the initial Wigner-Poisson equation.

\subsection{Full discretization}

Starting with the numerical method proposed in [5], we derive a numerical scheme for (2.14), in particular for the numerical resolution of $T_{\varepsilon h_{0}}[\phi]$.

Let us denote $x_{i}=i \Delta x, i=-1, \cdots, N_{x}$ and $v_{k}=v_{\min }+k \Delta v, k=0, \cdots, N_{v}-1$ where $\Delta x=L / N_{x}$ and $\Delta v=\left(v_{\max }-v_{\min }\right) / N_{v}$ (with $\left.v_{\max }=-v_{\min }\right)$ are the uniform phase space discretizations. Hence we denote by $g_{i, k}^{n}$ an approximation of $g\left(t^{n}, x_{i}, v_{k}\right)$ and $M_{k}=M\left(v_{k}\right)$. On the other hand, the macro part of (2.14) is approximated at $x_{i}=i \Delta x, i=0, \cdots, N_{x}$ so that we use the notations $\rho_{i}^{n} \approx \rho\left(t^{n}, x_{i}\right)$. The electric potential is evaluated at $x_{i}$ by $\phi_{i}\left(t^{n}\right)$ and we can reconstruct the total distribution function $f_{i, k}^{n}=\rho_{i}^{n} M_{k}+g_{i, k}^{n}$.

Recalling that $\mathcal{T}=v \partial_{x}-T_{\varepsilon h_{0}}\left[\phi^{n}\right]$, we use a finite volume scheme for the transport in the spatial variable to get the following approximation for $(\mathcal{T} f)\left(t^{n}, x_{i}, v_{j}\right)$ :

$$
\left(\mathcal{T} f^{n}\right)_{i, k}=v_{k}^{+} \frac{f_{i, k}^{n}-f_{i-1, k}^{n}}{\Delta x}+v_{k}^{-} \frac{f_{i+1, k}^{n}-f_{i, k}^{n}}{\Delta x}-T_{\varepsilon h_{0}}\left[\phi^{n}\right] f_{i, k}^{n}
$$


so that the numerical scheme for (4.27) reads as

$$
g_{i, k}^{n+1}\left(1+\frac{\varepsilon^{2}}{\Delta t}\right)=\frac{\varepsilon^{2}}{\Delta t} g_{i, k}^{n}-\varepsilon(I-\Pi)\left(\mathcal{T} f^{n}\right)_{i, k}
$$

Here the projection $\Pi f$ is approximated by the following discrete operator $(\Pi f)_{i, k} \approx\left(\sum_{k} f_{i, k} \Delta v\right) M_{k}$ whereas the integrals in $v$ are approximated by a sum on the discrete velocities. Once $g_{i, k}^{n+1}$ is computed, the time-stepping of the macro equation is

$$
\rho_{i}^{n+1}=\rho_{i}^{n}-\beta \frac{\Delta t}{\varepsilon}\left(\sum_{k}\left(\mathcal{T} f^{n}\right)_{i, k} \Delta v\right)-(1-\beta) \frac{\Delta t}{\varepsilon} \sum_{k}\left(v_{k} \frac{g_{i+1, k}^{n+1}-g_{i-1, k}^{n+1}}{2 \Delta x} \Delta v\right) .
$$

The Poisson equation can be discretized to get the potential at $x_{i}$.

$$
-\left[\phi_{i+1}^{n}-2 \phi_{i}^{n}+\phi_{i-1}^{n}\right]=\Delta x^{2}\left(\rho_{i}^{n}-1\right) .
$$

Finally, the Wigner term $T_{\varepsilon h_{0}}\left[\phi^{n}\right]$ is discretized using the spectral method described in Ref. [5].

The asymptotic property can also be proven in the full discretized context. Indeed, in the discrete micro equation for $g$, we get as $\varepsilon$ goes to zero

$$
\begin{aligned}
& g_{i, k}^{n+1}=-\varepsilon(I-\Pi)\left[v_{k}^{+} \frac{f_{i, k}^{n}-f_{i-1, k}^{n}}{\Delta x}+v_{k}^{-} \frac{f_{i+1, k}^{n}-f_{i, k}^{n}}{\Delta x}-T_{\varepsilon h_{0}}\left[\phi^{n}\right] f_{i, k}^{n}\right]+\mathcal{O}\left(\varepsilon^{2}\right), \\
& =-\varepsilon(I-\Pi)\left[M_{k} v_{k}^{+} \frac{\rho_{i}^{n}-\rho_{i-1}^{n}}{\Delta x}+M_{k} v_{k}^{-} \frac{\rho_{i+1}^{n}-\rho_{i}^{n}}{\Delta x}-T_{\varepsilon h_{0}}\left[\phi^{n}\right] f_{i, k}^{n}\right] \mathcal{O}\left(\varepsilon^{2}\right), \\
& =-\varepsilon\left[M_{k} v_{k}^{+} \frac{\rho_{i}^{n}-\rho_{i-1}^{n}}{\Delta x}+M_{k} v_{k}^{-} \frac{\rho_{i+1}^{n}-\rho_{i}^{n}}{\Delta x}\right] \\
& +\varepsilon \frac{\rho_{i}^{n}-\rho_{i-1}^{n}}{\Delta x} \Pi\left(M_{k} v_{k}^{+}\right)+\varepsilon \frac{\rho_{i+1}^{n}-\rho_{i}^{n}}{\Delta x} \Pi\left(M_{k} v_{k}^{-}\right)+\varepsilon T_{\varepsilon h_{0}}\left[\phi^{n}\right] f_{i, k}^{n}+\mathcal{O}\left(\varepsilon^{2}\right), \\
& =-\varepsilon\left[M_{k} v_{k}^{+} \frac{\rho_{i}^{n}-\rho_{i-1}^{n}}{\Delta x}+M_{k} v_{k}^{-} \frac{\rho_{i+1}^{n}-\rho_{i}^{n}}{\Delta x}\right] \\
& +\varepsilon \frac{\rho_{i}^{n}-\rho_{i-1}^{n}}{\Delta x} \Pi\left(M_{k}\left|v_{k}\right|\right) / 2-\varepsilon \frac{\rho_{i+1}^{n}-\rho_{i}^{n}}{\Delta x} \Pi\left(M_{k}\left|v_{k}\right|\right) / 2+\varepsilon T_{\varepsilon h_{0}}\left[\phi^{n}\right] f_{i, k}^{n}+\mathcal{O}\left(\varepsilon^{2}\right), \\
& =-\varepsilon\left[M_{k} v_{k}^{+} \frac{\rho_{i}^{n}-\rho_{i-1}^{n}}{\Delta x}+M_{k} v_{k}^{-} \frac{\rho_{i+1}^{n}-\rho_{i}^{n}}{\Delta x}\right] \\
& +\varepsilon \frac{2 \rho_{i}^{n}-\rho_{i+1}^{n}-\rho_{i-1}^{n}}{2 \Delta x} \Pi\left(M_{k}\left|v_{k}\right|\right) / 2+\varepsilon T_{\varepsilon h_{0}}\left[\phi^{n}\right] f_{i, k}^{n}+\mathcal{O}\left(\varepsilon^{2}\right), \\
& =-\varepsilon M_{k} v_{k} \frac{\rho_{i+1}^{n}-\rho_{i-1}^{n}}{2 \Delta x}+\varepsilon \frac{\rho_{i+1}^{n}-2 \rho_{i}^{n}+\rho_{i-1}^{n}}{2 \Delta x}(I-\Pi)\left(M_{k}\left|v_{k}\right|\right) \\
& +\varepsilon T_{\varepsilon h_{0}}\left[\phi^{n}\right] f_{i, k}^{n}+\mathcal{O}\left(\varepsilon^{2}\right) \text {. }
\end{aligned}
$$

But $(I-\Pi)\left(M_{k}\left|v_{k}\right|\right)=M_{k}\left(\left|v_{k}\right|-\sqrt{2 / \pi}\right)$, which is even, so that $v_{k}(I-\Pi)\left(M_{k}\left|v_{k}\right|\right)$ is odd and $\Pi\left(v_{k}(I-\Pi)\left(M_{k}\left|v_{k}\right|\right)\right)=0$. Then, injecting $g_{i, k}^{n+1}$ into (4.32) leads to

$$
\begin{aligned}
\frac{\rho_{i}^{n+1}-\rho_{i}^{n}}{\Delta t} & =\Pi\left(v_{k}^{2} M_{k}\right) \frac{\rho_{i+2}^{n}-2 \rho_{i}^{n}+\rho_{i-2}^{n}}{4 \Delta x^{2}}-\frac{\Pi\left(v_{k} T_{\varepsilon h_{0}}\left[\phi^{n}\right] f_{i+1, k}^{n}\right)-\Pi\left(v_{k} T_{\varepsilon h_{0}}\left[\phi^{n}\right] f_{i-1, k}^{n}\right)}{2 \Delta x}+\mathcal{O}(\varepsilon), \\
& =\partial_{x}\left[\partial_{x} \rho^{n}+\rho^{n} \partial_{x} \phi^{n}\right]+\mathcal{O}(\Delta x+\Delta v)+\mathcal{O}(\varepsilon),
\end{aligned}
$$

which is consistent with the diffusion equation (2.11).

Using staggered grids $x_{i+1 / 2}$ and a specific transport scheme for $\rho$, enables us to obtain the standard centered scheme for the second derivative in $(2.11):\left(\rho_{i+1}-2 \rho_{i}+\rho_{i-1}\right) / \Delta x^{2}$. These steps are fully detailed in [4]. 


\section{$5 \quad$ Numerical results}

We consider the following initial condition

$$
f(t=0, x, v)=\frac{1}{a}\left(\exp \left(-\frac{1}{2 \theta}|v-2|^{2}\right)+\exp \left(-\frac{1}{2 \theta}|v+2|^{2}\right)\right)(1+\alpha \varphi(x))
$$

where $a$ is a normalization factor, $\alpha=10^{-3}, \varphi(x)=\cos \left(k x+b_{j}\right), b_{j}$ being a random number between 0 and $2 \pi$, and $k$ is the wave number (here we use $k=0.2$ ). For the micro-macro model (2.14), the initial conditions read as

$$
\rho(t=0, x)=1+\alpha \varphi(x), \quad \text { and } \quad g(t=0, x, v)=f(t=0, x, v)-\rho(t=0, x) \frac{\exp \left(-v^{2} / 2\right)}{\sqrt{2 \pi}},
$$

whereas for the limit model $(2.11)$ we have

$$
\rho(t=0, x)=1+\alpha \varphi(x) .
$$

The numerical parameters are chosen as follows: $N_{x}=300, N_{v}=512$ (with two buffer zones of 25 points) and $\Delta t$ is chosen to respect the CFL condition $\Delta t<\Delta x / v_{\max }$, with $v_{\max }=20$. As to the physical parameters, the normalized Planck constant is fixed to $h_{0}=10^{-2}$, the temperature is either $\theta=1$ or $\theta=0.2$, and $\varepsilon$ spans a wide range of values, from $10^{-8}$ to $10^{4}$.

We are interested in three regimes: the diffusive regime $(\varepsilon \ll 1)$, the intermediate regime $[\varepsilon=\mathcal{O}(1)]$, and the collisionless transport regime $(\varepsilon \gg 1)$. We shall compare the micro-macro Wigner-BGK model (2.14), here denoted MMW, to either the drift-diffusion (DD) model (2.11) in the diffusive regime or to the full Wigner-BGK model (2.7) in the other regimes.

As a diagnostic tool, we consider the time history of the electric energy $\|E(t)\|_{L^{2}}^{2}$ (the integral of the square of the electric field) for the three models, in semi-log scale. In the collisionless regime, we also compare the numerical results to the analytical expression for the linear growth rate given in Sec. 3.

\subsection{Diffusive regime}

First, we consider very small values of $\varepsilon$. In Fig. 2, the electric energy is shown for the MMW (2.14) and DD (2.11) models, for $\varepsilon=10^{-2}, 10^{-4}$, and $10^{-8}$. For all three cases shown in Fig. 2, the time step for MMW is $\Delta t=5 \times 10^{-3}$, which is much larger than the time step required for simulating the full Wigner equation (2.7), which requires $\Delta t=\mathcal{O}\left(\varepsilon^{2}\right)$.

We notice that, for these values of $\varepsilon$, the diffusive regime is already attained, since the two curves (MMW and DD) are nearly superimposed. The observed damping of the electric energy is a consequence of the BGK relaxation operator, which acts to restore the equilibrium configuration $M_{\Theta}$.
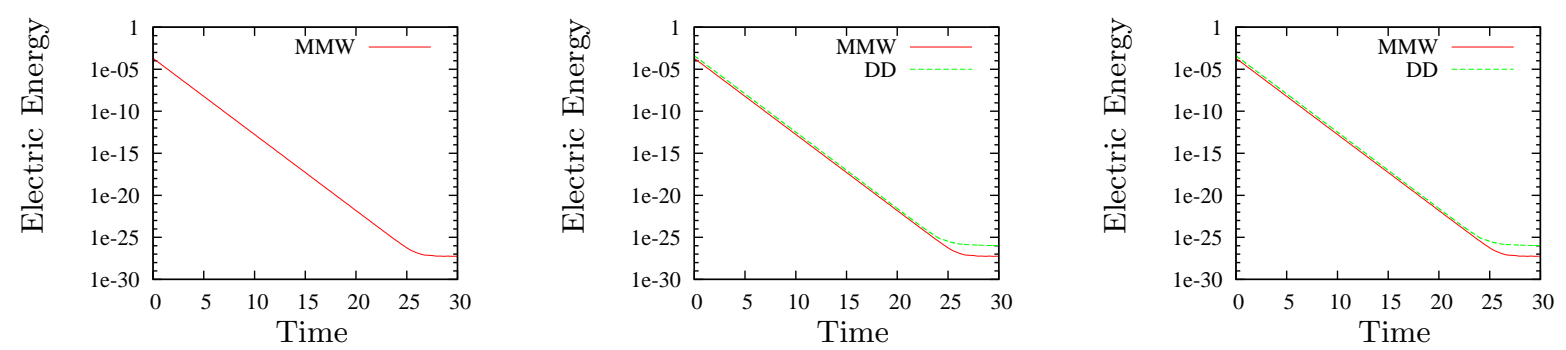

Figure 2: Diffusive regime. Time history of the electric energy for $\varepsilon=10^{-2}$ (left), $\varepsilon=10^{-4}$ (middle), and $\varepsilon=10^{-8}$ (right). 


\section{$5.2 \quad$ Intermediate regime}

Here, we are interested in the intermediate regimes $\varepsilon=\mathcal{O}(1)$, for which the original Wigner-BGK model (2.7) can be simulated and compared to MMW. The time step $\Delta t$ used for both MMW and Wigner-BGK is equal to $10^{-3}$ (so that the CFL condition $\Delta t<\Delta x / v_{\max }$ is satisfied).

The two models agree very well in this regime, as can be seen in Figs. 3 and 4 . For the MMW model, the damping of the electric energy continues for longer times compared to the Wigner-BGK model. This behaviour may be explained by the fact that the MMW code is less sensitive to round-off errors than the Wigner code.
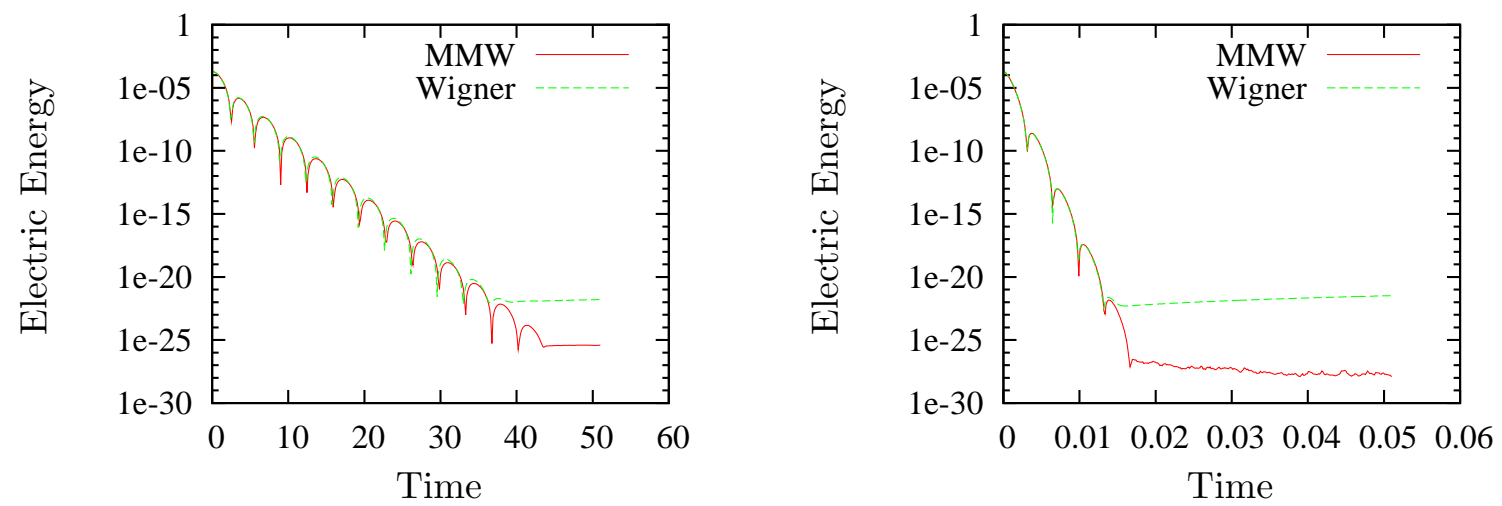

Figure 3: Intermediate regime. Time history of the electric energy for $\varepsilon=1$ (left) and $\varepsilon=0.6$ (right).
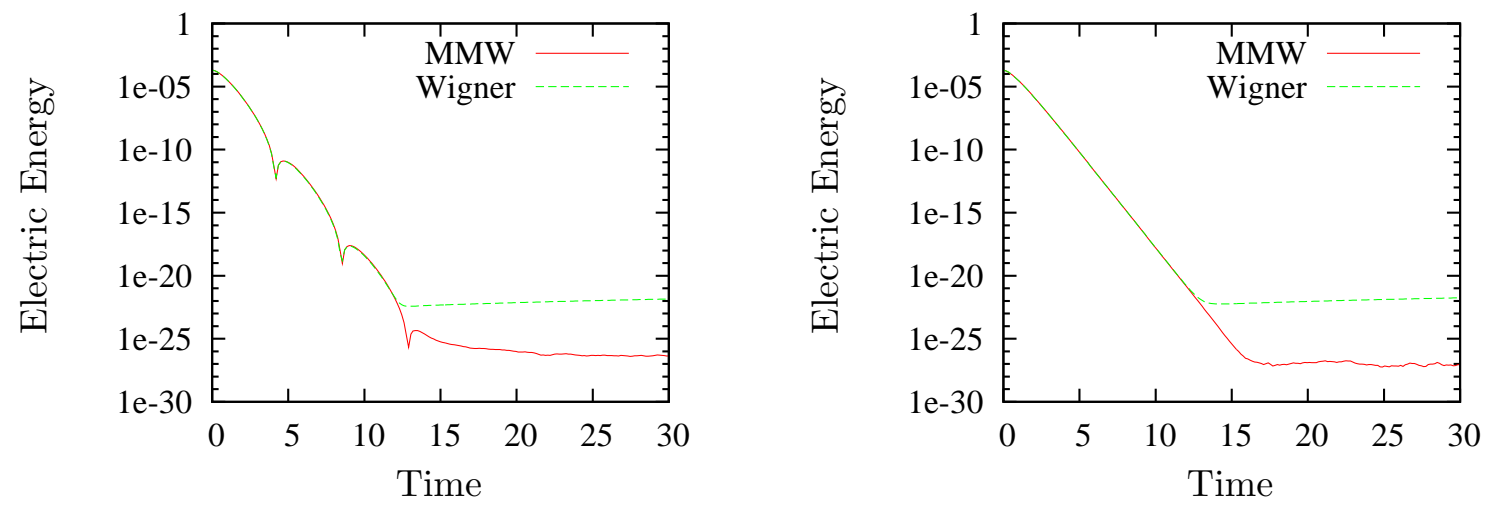

Figure 4: Intermediate regime. Time history of the electric energy for $\varepsilon=0.55$ (left) and $\varepsilon=0.5$ (right).

\subsection{Collisionless transport regime}

In this regime $\varepsilon \gg 1$. Thus, if we rescale the time $t$ by $t / \varepsilon$ in Eq. (2.7), we recover the standard collisionless Wigner-Poisson equations. We can therefore compare the numerical results to analytical estimates based on the linearization of the Wigner-Poisson equations (see Sec. 3).

In Fig. 5, we show the results for $\varepsilon=50$ and $\varepsilon=300$. Again, the MMW and Wigner models yield practically the same results. The electric energy is still damped, but the damping rate is smaller compared to the diffusive and intermediate regimes. This is due to a competition between two effects: (i) on the one hand, the BGK term tends to damp the electric energy with a rate going 
as $\varepsilon^{-1}$; (ii) on the other hand, the initial two-stream equilibrium is potentially unstable, which would lead to a growth of the electric energy. The net result is still damping, but with a lower rate.

Very large values of $\varepsilon\left(10^{3}\right.$ and $\left.10^{4}\right)$ are considered in Figs. 6 and 7 . Even in this regime, it is clear that the MMW code is capable of perfectly reproducing the Wigner-Poisson results. We note that, for $\varepsilon=10^{3}$, the electric energy grows exponentially during the early stages of the simulation, signalling an instability (left panel of Figs. 6 and 7). The instability is even more visible in the zooms of these plots, shown in Fig. 8. Indeed, if we plug the relevant parameters $(k=0.2, V=2$, $\left.h_{0}=0.01\right)$ in Eq. (3.25) for the linear frequency, we obtain $\Omega^{2}<0$, so that the frequency has a non-zero imaginary part. For instance, for $\varepsilon=10^{3}$ and $\theta=0.2$, one obtains $\operatorname{Im}(\Omega) / \varepsilon \approx 0.21$, which is in good agreement with the observed growth rate shown in the right panel of Fig. 8 (since the energy is a quadratic quantity in the electric field, the growth rate has to be multiplied by a factor two). For $\theta=0.2$ (left panel of Fig. 8) the agreement is also good.

Using the same parameters but taking $\varepsilon=10^{4}$ leads to a positive value of $\Omega^{2}$ in Eq. (3.25), so that the frequency is a real quantity and no instability should occur. This is confirmed by the right panel of Figs. 6 and 7, where we see that the electric energy is damped away.

Finally, we note that this regime is well reproduced by the MMW code thanks to the use of the "mixed scheme" detailed in Sec. 4, which makes it possible to recover asymptotically an explicit numerical scheme for the Wigner-Poisson equations.
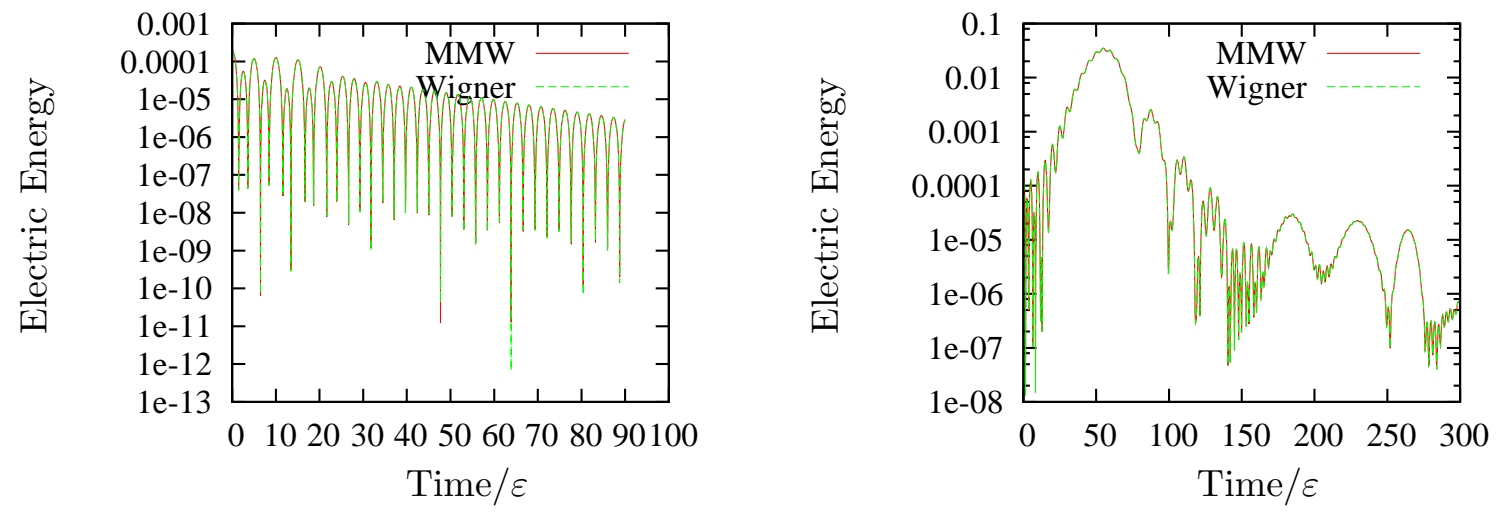

Figure 5: Collisionless transport regime. Time history of the electric field for $\varepsilon=50$ (left frame) and $\varepsilon=300$ (right frame).
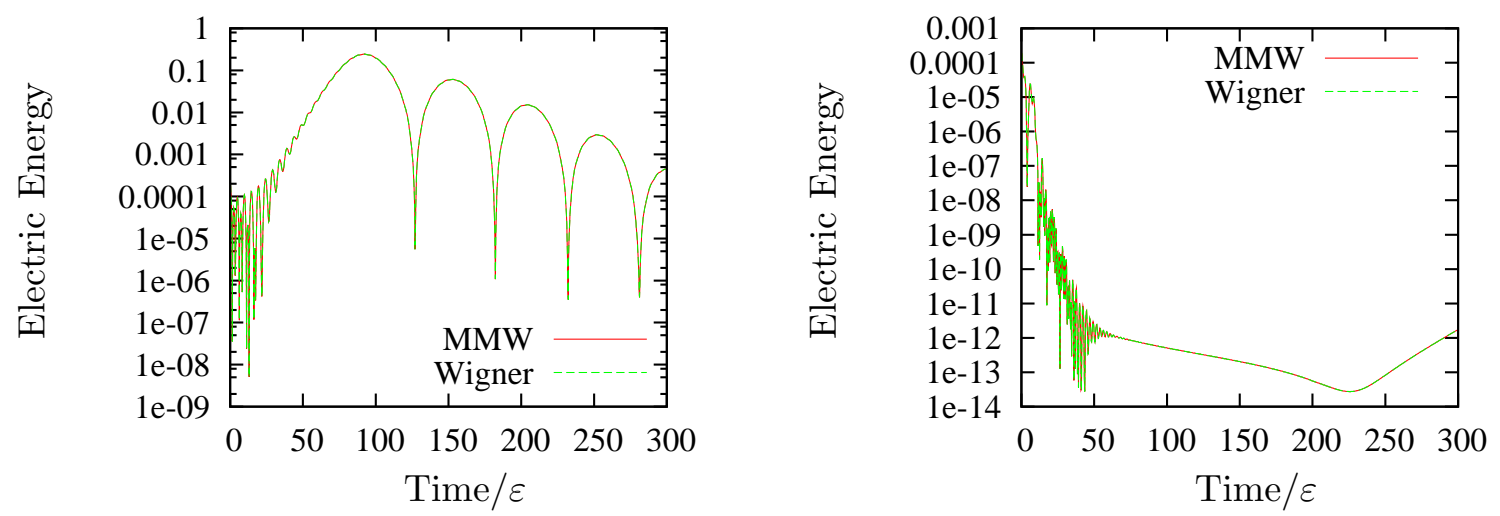

Figure 6: Collisionless transport regime. Time history of the electric energy for $\theta=1, \varepsilon=10^{3}$ (left frame) and $\varepsilon=10^{4}$ (right frame). 

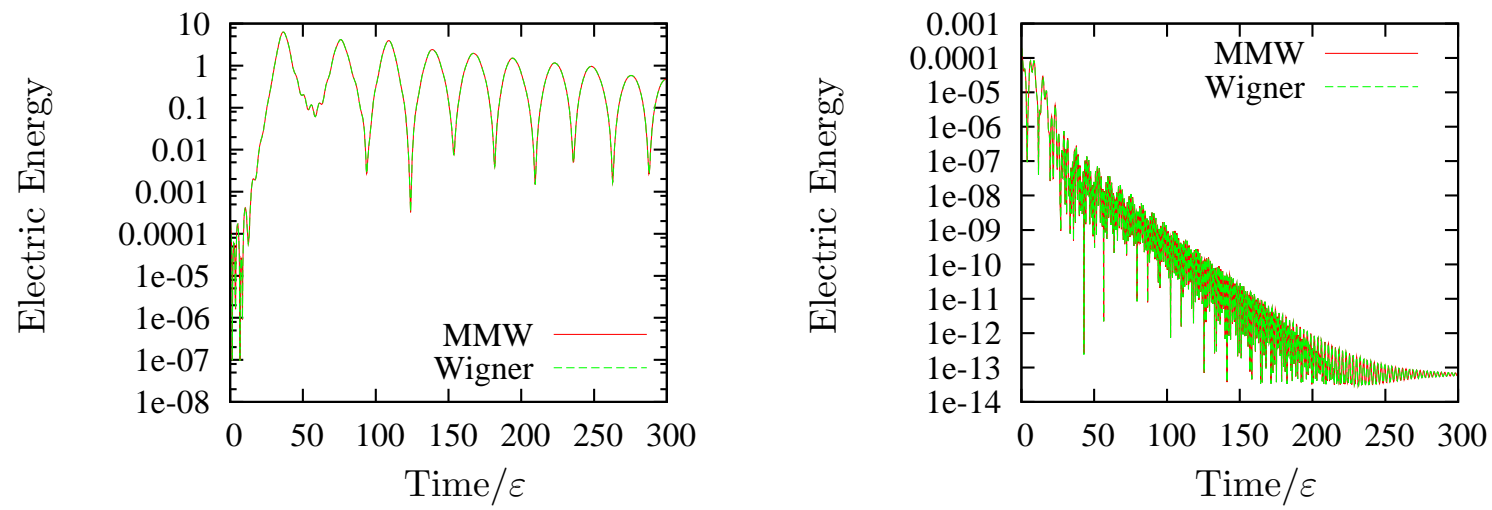

Figure 7: Collisionless transport regime. Time history of the electric energy for $\theta=0.2, \varepsilon=10^{3}$ (left frame) and $\varepsilon=10^{4}$ (right frame).
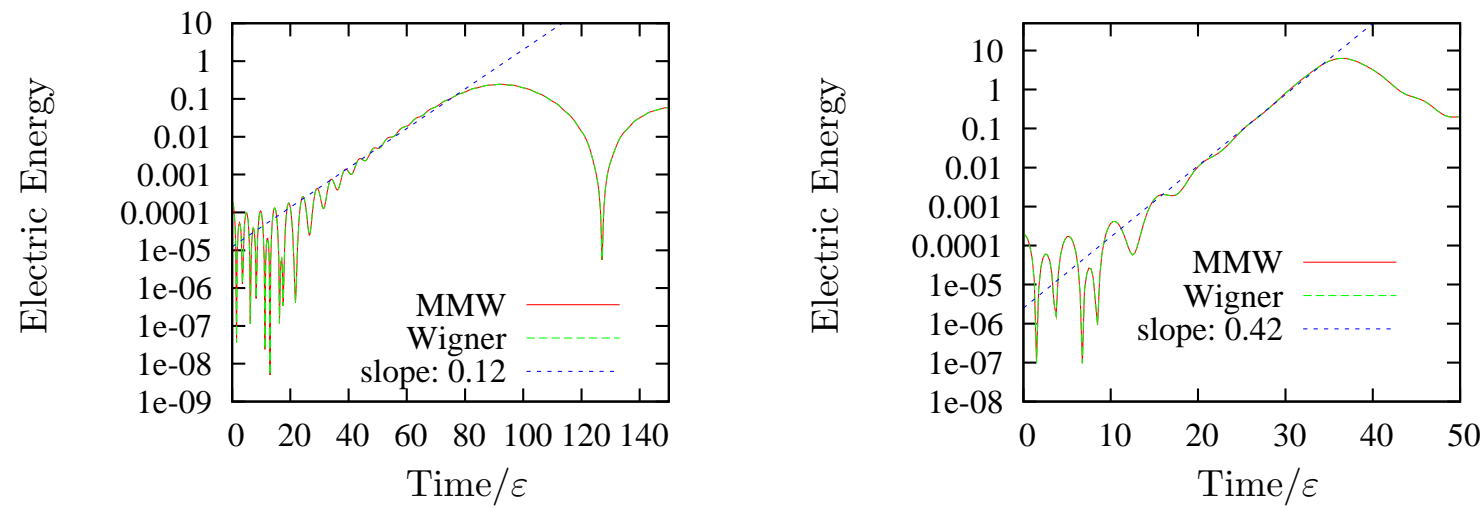

Figure 8: Zoom of the left frames in Figs. 6 and 7. $\varepsilon=10^{3}$ and $\theta=1$ (left frame); $\varepsilon=10^{3}$ and $\theta=0.2$ (right frame). The slope of the dashed straight lines corresponds to the instability rate multiplied by two.

\subsection{Position-dependent $\varepsilon(x)$}

In this paragraph, we consider the micro-macro Wigner-BGK model (2.14) in which the smallness parameter $\varepsilon$ depends on the spatial variable $x$, so that several regimes can coexist in the same simulation. The Wigner equation (2.7) should then be rewritten in the conservative form

$$
\frac{\partial f}{\partial t}+v \partial_{x}\left(\frac{1}{\varepsilon(x)} f\right)-\frac{1}{\varepsilon(x)} T_{\varepsilon(x) h_{0}}[\phi] f=\frac{1}{\varepsilon^{2}(x)}\left(\rho M_{\Theta}-f\right) .
$$

This type of problem has already been investigated in the hydrodynamic regime [10]. A similar profile for $\varepsilon(x)$ is considered here

$$
\varepsilon(x)=\varepsilon_{0}+\varepsilon_{\max } \exp \left(-\frac{|x-\pi / k|^{2}}{4}\right), x \in[0,2 \pi / k],
$$

where $k=0.2, \varepsilon_{0}$ is a threshold value for $\varepsilon(x)$, and $\varepsilon_{\max }$ can in principle be very large. This spatial profile of $\varepsilon(x)$ represents a system where a collisionless region is surrounded by two strongly collisional buffer zones. 
The micro-macro model is well suited for this kind of situation, since the time step $\Delta t$ is chosen to respect the CFL condition $\Delta t<\Delta x / v_{\max }$, whereas for the full Wigner model one needs to take $\Delta t<\varepsilon_{0}^{2}$, which can be too restrictive when $\varepsilon_{0}$ is small.

Two cases will be considered here. First, we set $\varepsilon_{0}=0.1$ and $\varepsilon_{\max }=10$, so that comparisons between the MMW and Wigner models can still be performed (we use $\Delta t=10^{-3}$ for both schemes). In a second case, we shall take $\varepsilon_{0}=10^{-7}$ and $\varepsilon_{\max }=10^{3}$. For this value of $\varepsilon_{0}$, Wigner simulations are not feasible and therefore only the MMW results will be shown, which are obtained with a time step $\Delta t=5 \times 10^{-4}$.

We use the same initial condition (5.34)-(5.35) as in the cases with constant $\varepsilon$ and the number of points in the phase space is $N_{x}=200, N_{v}=256$. We are interested in the density $\rho(x)$ as well as the full distribution function $f(x, v)$ at different times [for the MMW scheme, the distribution function is $\left.f_{\mathrm{MMW}}=\rho(x) \exp \left(-v^{2} / 2\right) / \sqrt{2 \pi}+g(x, v)\right]$.

In Figs. 9, 10 and 11, we plot the phase-space distribution function at times $t=0.1,2$ and 10 , obtained with the Wigner and MMW schemes, for the case $\varepsilon_{0}=0.1$ and $\varepsilon_{\max }=10$. For early times, a vortex is created in the middle of the domain, where the plasma is essentially collisionless. Outside this domain the plasma quickly becomes strongly collisional, so that the formation of phase space structures is inhibited. The transition to the diffusive regime occurs around $x=10$ and $x=20$. Even in the middle of the domain, the collisions bring the system back to equilibrium for later times $(t=20)$.

We stress that the phase space plots obtained from the Wigner and MMW models are virtually identical. The good behavior of the MMW scheme is emphasized in Fig. 12, where the densities $\rho(x)$ obtained with the two methods are displayed at $t=1$.
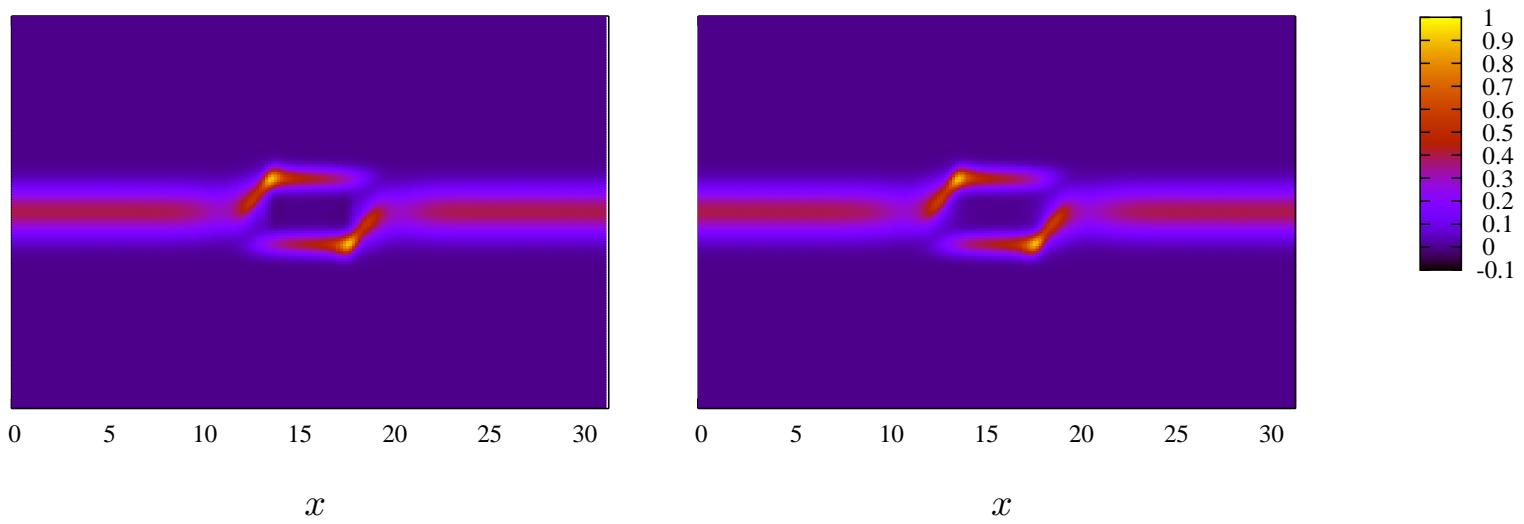

$x$

Figure 9: Phase space distribution function $f(x, v)$ at time $t=0.1$. Left frame: MMW; right frame: Wigner. 

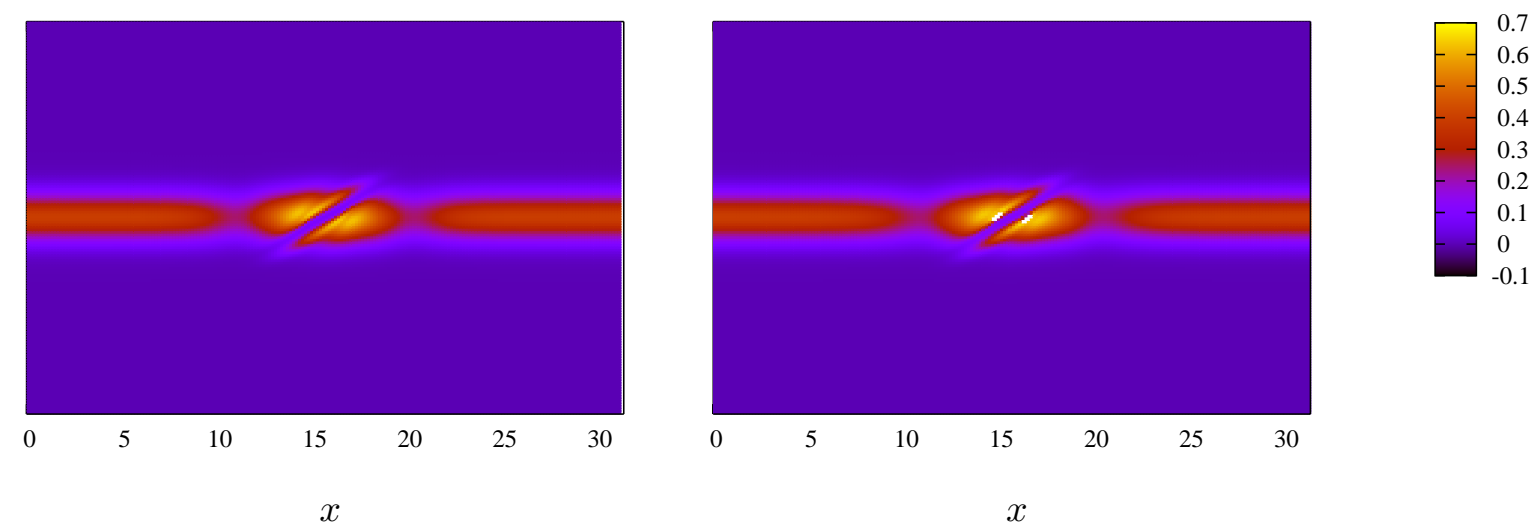

Figure 10: Phase space distribution function $f(x, v)$ at time $t=2$. Left frame: MMW; right frame: Wigner.
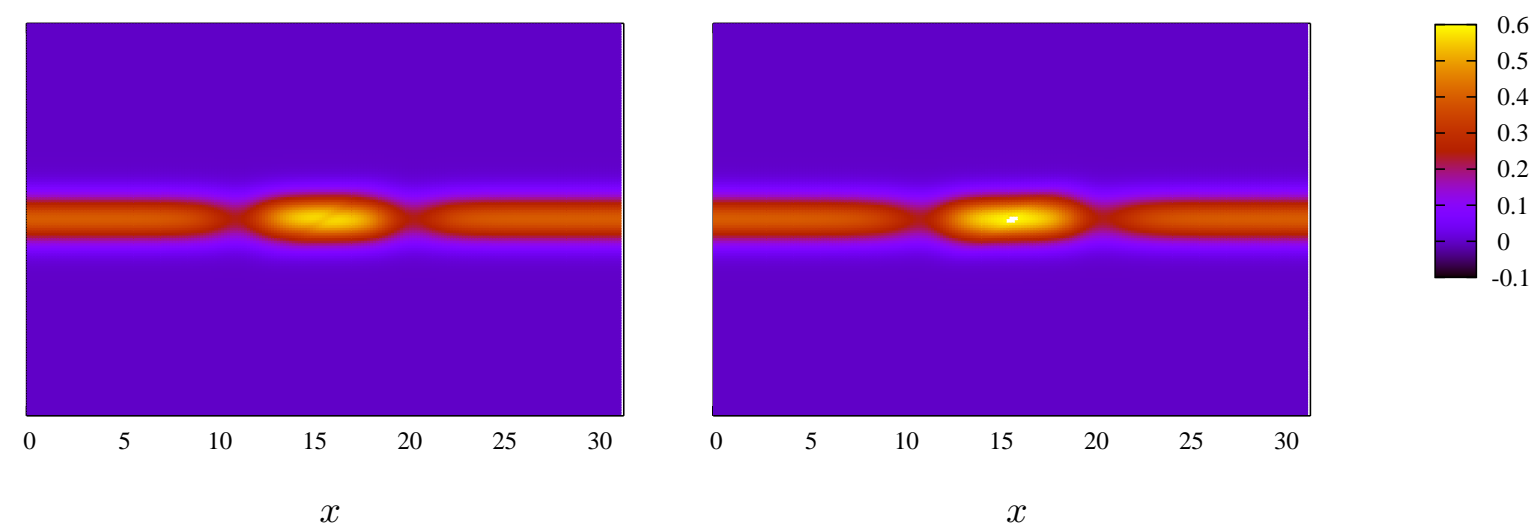

Figure 11: Phase space distribution function $f(x, v)$ at time $t=10$. Left frame: MMW; right frame: Wigner. 


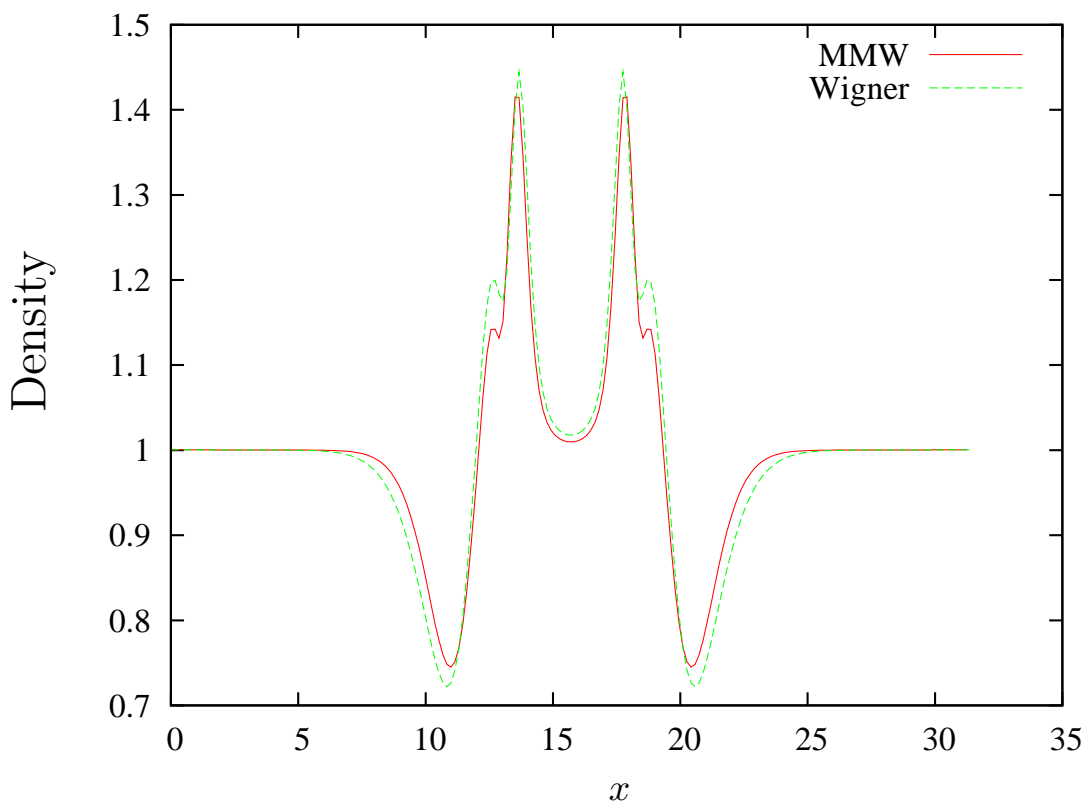

Figure 12: Spatial density $\rho(x)$ at time $t=1$ obtained with the MMW scheme (solid red line) and the Wigner scheme (dashed green line).

For the second run, we take $\varepsilon_{0}=10^{-7}$ and $\varepsilon_{\max }=10^{3}$. In this case, the Wigner scheme cannot be used as it would require a time step $\Delta t \sim \varepsilon_{0}^{2}$. Hence, only numerical results obtained with the MMW method are shown. In Fig. 13, the distribution function $f(x, v)$ is plotted at time $t=1$, whereas the spatial density $\rho(x)$ is displayed in Fig. 14. As in the previous case, a transition zone - separating the diffusion regime from the collisionless regime - can be clearly identified around $x=10$ and $x=20$.

Note that this test is rather challenging, since the numerical scheme must be capable of handling two very disparate regimes at the same time. An alternative strategy in which a diffusion model such as Eq. (2.11) is used in the small $\varepsilon$ region and a Wigner model is used in the large $\varepsilon$ region would require some carefully tailored boundary conditions at the interface between the two subdomains, which is usually a very delicate task. 


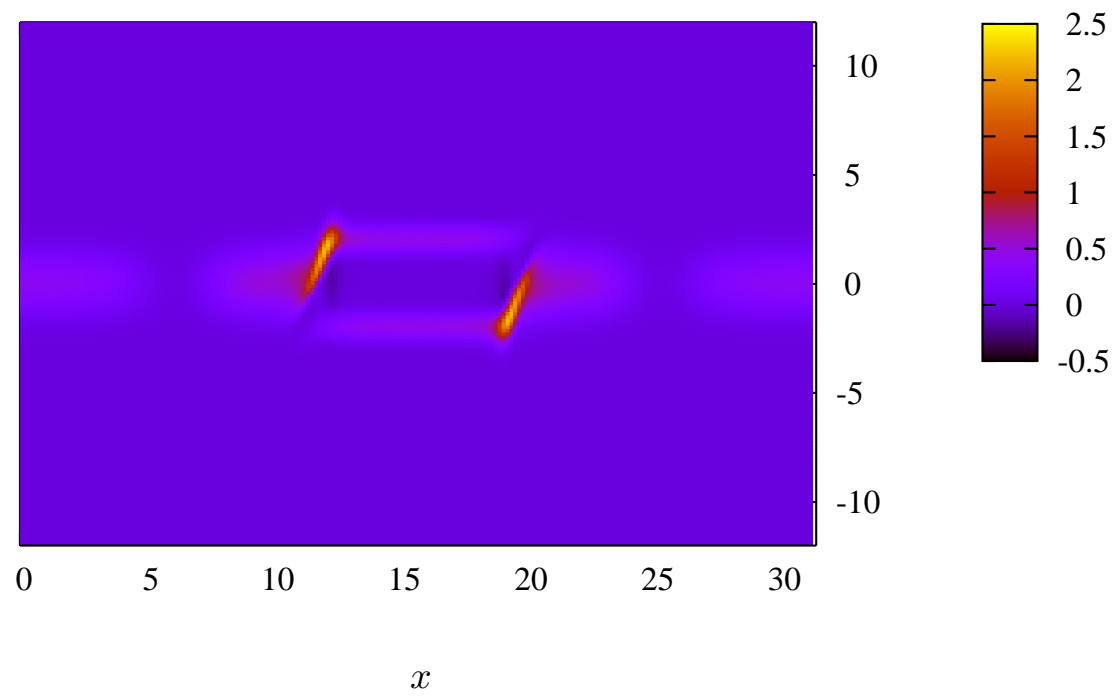

Figure 13: Distribution function at time $t=1$ obtained with the MMW scheme: $f_{\mathrm{MMW}}(t=$ $1, x, v)=\rho(t=1, x) \exp \left(-v^{2} / 2\right) / \sqrt{2 \pi}+g(t=1, x, v)$.

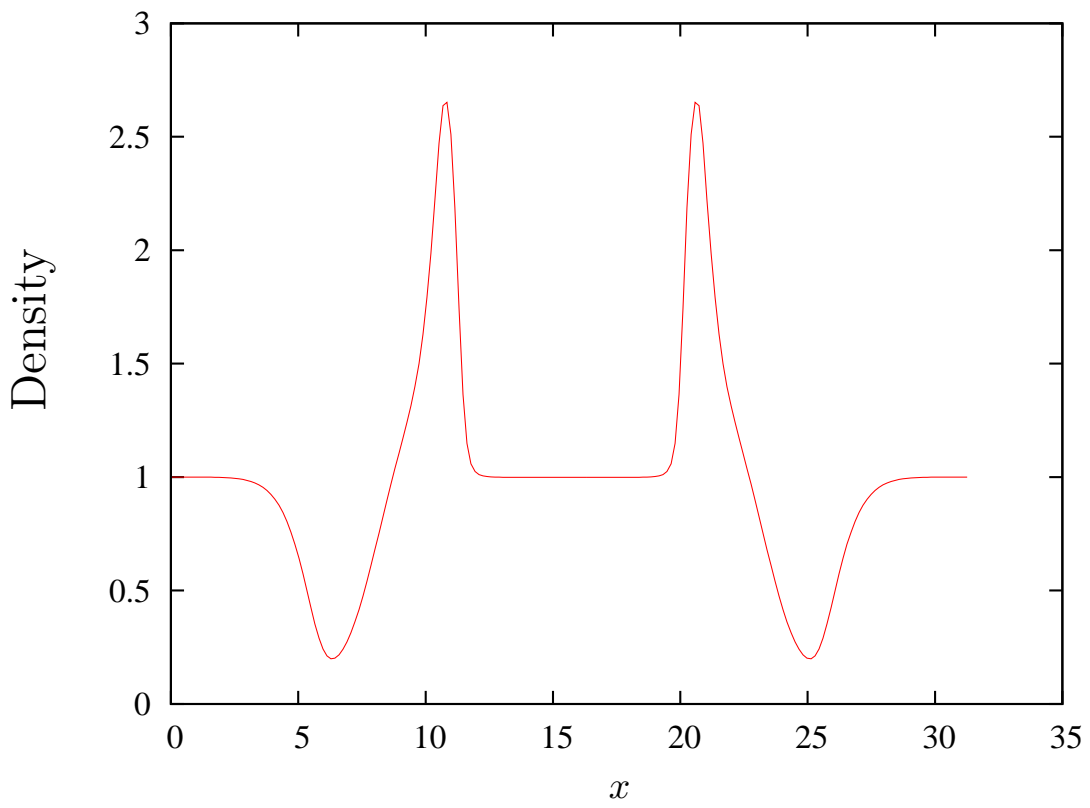

Figure 14: Spatial density $\rho(x)$ at time $t=1$ obtained with the MMW scheme. 


\section{Conclusion}

In this work, we developed a micro-macro numerical scheme for the Wigner-Poisson-BGK equation (named MMW scheme), which satisfies the following asymptotic properties: (i) when $\varepsilon \rightarrow 0$, it reduces to a consistent discretization of the drift-diffusion equation, for a fixed set of numerical parameters that is not restricted by $\varepsilon$; (ii) when $\varepsilon \rightarrow+\infty$, it reduces to the original explicit discretization of the collisionless Wigner-Poisson equation.

Numerical tests have proven that the MMW scheme reproduces correctly the results of the drift-diffusion model in the limit $\varepsilon \rightarrow 0$ and those of the Wigner-Poisson model when $\varepsilon=O(1)$ or $\varepsilon \gg 1$. In addition, when $\varepsilon$ is very large, the numerical computations agree with the analytical calculations of the linear instability rates.

Finally, we studied the case of a spatially dependent $\varepsilon$, for which this approach is well suited, since no domain decomposition is required and the AP scheme can be applied to the whole domain using a fixed set of numerical parameters. Again, the comparison between the MMW and Wigner schemes yielded very satisfactory results.

Several extensions of the present work may be envisaged. More physically realistic collision operators (e.g., Fokker-Planck) could be considered and the present strategy could be extended along the ideas presented in [23]. It would also be interesting to use higher order numerical schemes for the phase space discretization of the micro-macro model.

\section{References}

[1] A. Arnold, J.-A. Carrillo, I. Gamba, C.-W. Shu, Low and high-field scaling limits for the Vlasov- and Wigner-Poisson-Fokker-Planck system, Transport Theory Statist. Phys. 30, pp. 121-153, (2001).

[2] N. W. Ashcroft, N. D. Mermin, Solid State Physics (Harcourt Brace, Orlando, 1976)

[3] P. L. Bhatnagar, E. P. Gross, M. Krook, A Model for Collision Processes in Gases. I. Small Amplitude Processes in Charged and Neutral One-Component Systems, Phys. Rev. 94, pp. 511-525, (1954).

[4] M. Bennoune, M. Lemou, L. Mieussens, Uniformly stable numerical schemes for the Boltzmann equation preserving the compressible Navier-Stokes asymptotics, J. Comput. Phys. 227, pp. 3781-3803, (2008).

[5] N.-D. Suh, M. R. Feix, P. Bertrand, Numerical simulation of the quantum LiouvillePoisson system, J. Comput. Phys. 94, pp. 403-418, (1991).

[6] C. Buet, S. Cordier, Numerical analysis of conservative and entropy schemes for the FokkerPlanck-Landau equation, SIAM J. Numer. Anal. 36, pp. 953-973, (1998).

[7] J. A. Carrillo, T. Goudon, P. Lafitte, F. Vecil, Numerical schemes of diffusion asymptotics and moment closures for kinetic equations, J. Sci. Comput. 36, pp. 113-149, (2008).

[8] N. Crouseilles, M. Lemou, An asymptotic preserving scheme based on a micro-macro decomposition for collisional Vlasov equations: diffusion and high-field scaling limits, to appear in Kinetic Related Models.

[9] P. Degond, B. Lucquin-Desreux, An entropy scheme for the Fokker-Planck collision operator in the Coulomb case, Numer. Math. 68, pp. 239-262, (1994). 
[10] F. Filbet, S. Jin, A class of asymptotic preserving schemes for kinetic equations and related problems with stiff sources, J. Comp. Phys. 229, pp. 7625-7648, (2010).

[11] R. Jasiak, G. Manfredi, P.-A. Hervieux, M. Haefele, Quantum-classical transition in the electron dynamics of thin metal films, New J. Phys. 11, 063042 (2009).

[12] M. Hillery, R.F. O'Connell, M.O. Scully, E.P. Wigner, Distribution functions in physics: Fundamentals, Phys. Rep. 106, pp. 121-167 (1984).

[13] S. Jin, Efficient asymptotic-preserving (AP) schemes for some multiscale kinetic equations, SIAM J. Sci. Comput. 21, pp. 441-454, (1999).

[14] S. Jin, D. Levermore, The discrete-ordinate method in diffusive regimes, Transport Theory Stat. Phys. 22, pp. 739-791, (1993).

[15] S. Jin, D. Levermore, Numerical schemes for hyperbolic conservation laws with stiff relaxation terms, J. Comput. Phys. 126, pp. 449-467, (1996).

[16] S. Jin, L. Pareschi, G. Toscani, Uniformly accurate diffusive relaxation schemes for multiscale transport equations, SIAM J. Num. Anal. 38, pp. 913-936, (2000).

[17] S. Jin, L. WAnG, An asymptotic preserving scheme for the Vlasov-Poisson-Fokker-Planck system in the high field regime, Acta Mathematica Scientia 31, pp. 2219-2232, (2011).

[18] A. KlaR, Asymptotic-induced domain decomposition methods for kinetic and drift diffusion semiconductors equations, SIAM J. Numer. Anal. 19, pp. 2032-2050, (1998).

[19] A. KLAR, An asymptotic-induced scheme for nonstationary transport equations in the diffusive limit, SIAM J. Numer. Anal. 35, pp. 1073-1094, (1998).

[20] A. Klar, A Numerical Method for Kinetic Semiconductor Equations in the Drift Diffusion Limit, SIAM J. Sci. Comp. 20, pp. 1696-1712, (1999).

[21] A. Klar, C. Schmeiser, Numerical passage from radiative heat transfer to nonlinear diffusion models, Math. Models Methods Appl. Sci. 11, pp. 749-767, (2001).

[22] N.C. Kluksdahl, A.M. Kriman, D.K. Ferry, C. Ringhofer, Phys. Rev. B 39, pp. 7720-7735 (1989).

[23] M. Lemou, Relaxed micro-macro schemes for kinetic equations, Comptes Rendus Mathématique 348, pp. 455-460, (2010).

[24] M. Lemou, L. MÉHats, Micro-macro schemes for kinetic equations including boundary layers, to appear in SISC, SIAM J. on Scientific Computing.

[25] M. Lemou, L. Mieussens, A new asymptotic preserving scheme based on micro-macro formulation for linear kinetic equations in the diffusion limit, SIAM J. Sci. Comp. 31, pp. 334-368, (2008).

[26] G. Manfredi, P.-A. Hervieux, Autoresonant control of the many-electron dynamics in nonparabolic quantum, Appl. Phys. Lett. 91, 061108 (2007).

[27] P.A. Markowich, C.A. Ringhofer, C. Schmeiser, Semiconductor equations (Springer, Vienna, 1990).

[28] E.P. Wigner, On the Quantum Correction For Thermodynamic Equilibrium, Phys. Rev. 40, pp. 749-759 (1932). 